\title{
Turning Drops into Bubbles: Cavitation by Vapor Diffusion through Elastic Networks
}

\author{
M. A. Bruning $\odot,{ }^{1, *}$ M. Costalonga, ${ }^{2}$ J. H. Snoeijer, ${ }^{1}$ and A. Marin $\oplus^{1}$ \\ ${ }^{1}$ Physics of Fluids Group, Faculty of Science and Technology, Mesa+ Institute, \\ University of Twente, 7500 AE Enschede, Netherlands \\ ${ }^{2}$ Department of Mechanical Engineering, Massachusetts Institute of Technology, Cambridge, Massachusetts 02139, USA
}

(Received 23 December 2018; revised manuscript received 19 February 2019; published 19 November 2019)

\begin{abstract}
Some members of the vegetal kingdom can achieve surprisingly fast movements making use of a clever combination of evaporation, elasticity, and cavitation. In this process, enthalpic energy is transformed into elastic energy and suddenly released in a cavitation event which produces kinetic energy. Here, we study this unusual energy transformation by a model system: A droplet in an elastic medium shrinks slowly by diffusion and eventually transforms into a bubble by a rapid cavitation event. The experiments reveal the cavity dynamics over the extremely disparate timescales of the process, spanning 9 orders of magnitude. We model the initial shrinkage as a classical diffusive process, while the sudden bubble growth and oscillations are described using an inertial-(visco)elastic model, in excellent agreement with the experiments. Such a model system could serve as a new paradigm for motile synthetic materials.
\end{abstract}

DOI: 10.1103/PhysRevLett.123.214501

Unlike animal cells, the swelling and shrinkage of plant cells are fundamental for the motion of the whole body of the plant. The key is the wall surrounding the cells: a thin but stiff wall that allows the cells to sustain large pressure differences [1]. Motion in plants often occurs over extremely separated timescales, one in the range of hours and/or days, related with tissue swelling or shrinkage and the other in the order of fractions of milliseconds, related with mechanical or thermodynamics instabilities. This fast motion typically involves the storage of elastic energy in the system and its quick (and often dramatic) release. One example of such quick release of energy is triggering by elastic instabilities, like the snapping of the Venus flytrap [2]. It can also involve more violent phenomena as cavitation, as in the fern sporangia [3]: The fern's cells shrink when they dehydrate. This induces a deformation of the sporangium, which results in accumulation of elastic energy. This energy is then quickly released in a sudden cavitation event inside several of the fern's cells, which restores the elastic energy stored in the fern and catapults the spores at large distances [3]. A synthetic analog system was studied in the past [4] by cutting out cavities inside water-soaked hydrogels, while others have instead used a laser into hydrogels to generate a cavity, soaked it in water, and then left it to dehydrate $[5,6]$. In such examples, the hydrogel's high liquid affinity and rigidity yielded relatively small deformations which did not result in a significant release of mechanical energy.

In this Letter, we consider cavitation through evaporation of a drop in a soft gel [7] putting special emphasis in the cavitation and bubble expansion in the elastic medium. This system serves as a mechanical analog of a plant cell, where the use of large elastic deformations provides an attractive route for the conversion of elastic to mechanical energy. After a slow diffusion-limited droplet shrinkage in which elastic energy is stored in the material, a rapid cavitation event follows, releasing the energy suddenly. This system allows us to monitor for the first time the whole dynamics of such complex process, from the day-long fading of the liquid droplet, to the millisecond birth and growth of the cavitation bubble. Moreover, we show that these features are accurately captured by a modified Rayleigh-Plesset equation, accounting for the viscoelastic properties of the medium.

The system under study is a single millimetric water droplet trapped in an elastic medium. The gel used is Dow Corning poly(dimethylsiloxane) (PDMS) Sylgard184 mixed in a 1:10 ratio (curing agent:base polymer). The static shear modulus $\mu_{0}$ of this gel is $0.7 \mathrm{MPa}$, as measured using a rheometer (Anton Paar MCR 502). Figure 1(a1) shows an image of the droplet in its initial state. A transparent containing box is filled with uncured PDMS and placed inside a chamber with a Peltier temperature control unit (DataPhysics Instruments) at $70^{\circ} \mathrm{C}$. After an initial precuring period of the gel of 15 min a water droplet is inserted in the center of the box, either using a micropipette or a tapered capillary, depending on the desired initial droplet radius $R_{0}$. The injection of the droplet at this moment allows us to locate the droplet steadily at a given location, while the polymer structure is still not fully reticulated, avoiding the presence of an initial stress field in the PDMS. This experimental setup has been specifically designed to capture both the whole droplet shrinkage process (spanning approximately a day) and the final cavitation event (which occurs in milliseconds) in one single experiment with good temporal and spatial 


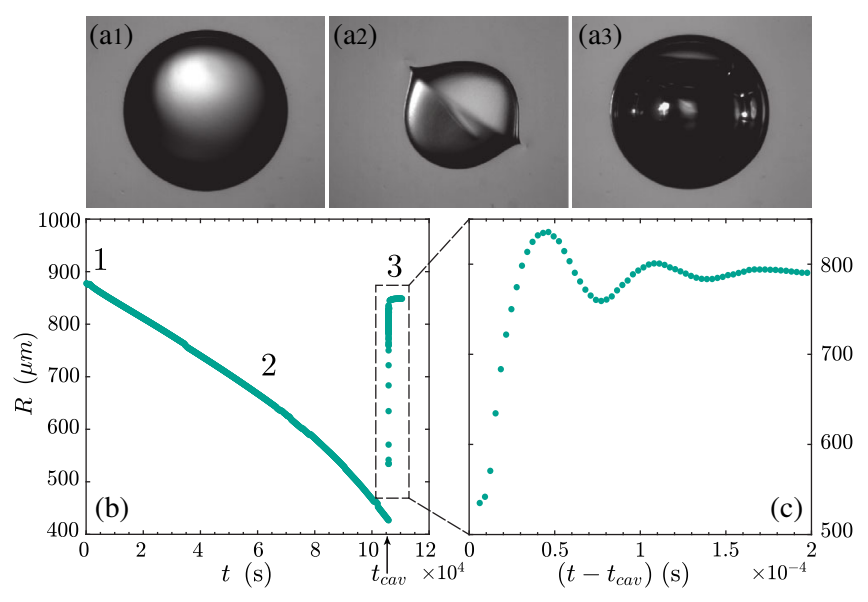

FIG. 1. Different phases in the life of a droplet in a dry and elastic medium. (a1) The initial water drop in a PDMS-based gel starts to shrink isotropically by evaporation. (a2) While shrinking, the elastic medium is deformed, certain amount of elastic energy is released by creasing at the solid-liquid interface. (a3) Eventually, enough negative elastic pressure is accumulated and a cavitation event occurs: A bubble of approximately the same size as the original liquid droplet is created. (b) The curve shows the evolution of the effective droplet radius in time and illustrates the disparity of timescales, from a day to a fraction of a millisecond. The corresponding instants for the images in the (a) panels are also indicated. (c) An enlargement of the cavity radius dynamics just after cavitation occurred at $t_{\text {cav }}$. A movie of the full droplet evaporation is given in the Supplemental Material [8].

resolution. To this end, we use two side view cameras, one recording at one frame per minute (Ximea MQ013MG$\mathrm{ON}$ ) and a high-speed camera (Photron SAX-2), mounted in the perpendicular direction. The high-speed camera is installed to capture the dynamics of the cavitation event, at the final stage of the experiment. Image triggering is used to start recording at frame rates in a range from 360 to $450 \mathrm{kfps}$. The duration of the full experiment varies from 3 to $50 \mathrm{~h}$ depending on the initial drop radius $R_{0}$ (from 200 to $1000 \mu \mathrm{m})$.

A typical experiment exhibits three stages, as shown in Fig. 1(a). At first, the droplet shrinks isotropically due to diffusion of water vapor into the surrounding PDMS [Fig. 1(a1)]. Then, at a certain time the droplet looses its spherical shape due to an elastic instability [Fig. 1(a2)]. During evaporation of the water droplet, the droplet size decreases and elastic stress builds up at the interface with the deformed material. Eventually, such stress is partially released by the formation of surface folds, triggering the socalled creasing instability [9-12]. In a somewhat different setup, Milner et al. [7] studied the onset of creasing for droplets immersed in gels. In the present work, creases are observed below a critical radius $R / R_{0}=0.73 \pm 0.05$, in agreement with Ref. [7]. Finally, as the droplet further evaporates, a negative pressure builds up inside the drop due to the tensile stresses exerted on the cavity, ultimately leading to cavitation [Fig. 1(a3)].
The final radius of the bubble is close to the initial droplet radius $\left(0.98 R_{0}\right)$, such that plastic deformation is negligible. The bubble in our study remains stable after the cavitation event occurs. This differs from the behavior of laser-induced bubbles in air-saturated hydrogels [13], in which gas diffusion leads to further bubble growth. An example of how the radius $R(t)$ typically evolves over time is provided in Fig. 1(b). After the creasing, the droplet is no longer spherical and we characterize its size with an effective radius using its projected area. The shrinking process is not altered substantially by the breaking of spherical symmetry, illustrated by the smoothness of $R(t)$ in Fig. 1(b). Finally, the data captured during the cavitation event are blown up in Fig. 1(c). A key feature of the experiment is the disparity of timescales in this single experiment, which span 9 orders of magnitude.

We now proceed with a more detailed analysis of each of the stages, starting with the slow droplet shrinkage. We hypothesize that this is a diffusion-limited evaporation process in thermal equilibrium, since water and PDMS have low affinity and no convection is present in the system. Rewriting the droplet radius $R$ as a function of time $t$, this predicts $R_{0}{ }^{2}-R^{2}=2 D \Delta c t / \rho$ [14]. Here, $R_{0}$ represents the initial droplet size, $\rho$ the density of water in its liquid phase, $D$ the diffusivity of the water vapor in the gel, and $\Delta c=c^{*}-c_{\infty}$ the concentration gradient between the interface and the far field (the last two properties are temperature dependent). The inset in Fig. 2 shows the typical change in radius $R_{0}^{2}-R^{2}$ versus time for different experimental conditions. The linear trend over a wide range of temperatures and ambient humidities (not shown here) confirms the diffusive behavior.

To quantitatively test the diffusion model, we represent the data in dimensionless form by scaling the radius as $R / R_{0}$ and time as $t^{*}=t / t_{F}=t 2 D \Delta c /\left(\rho_{w} R_{0}^{2}\right)$. For the

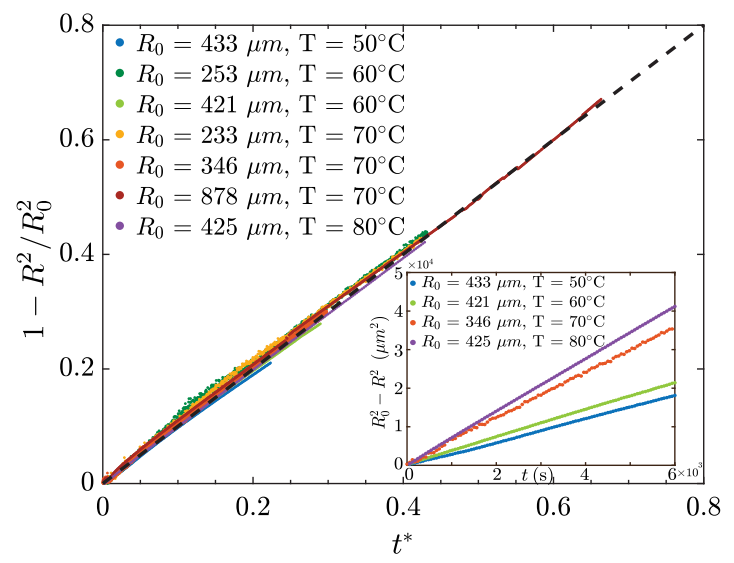

FIG. 2. Evaporation of a water droplet in PDMS: The plot shows the linear trend of the dimensionless droplet area loss $1-R^{2} / R_{0}^{2}$ against the dimensionless time $t^{*}=t / t_{F}$, for different initial radii and temperatures. The inset shows the same data in dimensional units. 
latter scaling, we take the diffusivity $D$ at different temperatures from [15], while $\Delta c$ is obtained by fitting the slopes in the inset in Fig. 2. Following this approach we achieve a collapse of the data, shown in the main panel, with values $\Delta c=40-50 \mathrm{~mol} / \mathrm{m}^{3}$. These are in good agreement with data from [16]. Hence, we conclude that the shrinkage of the droplet inside this elastic material is governed by a relatively simple diffusion-limited process.

The evaporation of the water droplet eventually leads to a very rapid cavitation event, the final stage in the droplet's life, at typical values of $R / R_{0}=0.48 \pm 0.1$. The exact location of the bubble nucleation is hard to determine from the images, but it seems to occur often at the liquid-gel interface. Since cavitation occurs here due to the tensile elastic stress that builds up in the gel, we estimate it by computing the elastic energy for a spherical cavity, which can be written as $\epsilon_{e l}=4 \pi \mu R_{0}^{3} F(\xi)$. In this expression $\mu$ is the shear modulus, $\xi=R / R_{0}$ the scaled bubble radius, and for a neo-Hookean solid the dimensionless function is $F(\xi)=\frac{5}{6} \xi^{3}-\xi^{2}-\frac{1}{3}+\frac{1}{2 \xi}[17,18]$. The minimal energy appears at $\xi=1$, at which the elastic medium is undeformed with respect to its reference state, and a tensile elastic stress appears for $\xi<1$. The (negative) pressure inside the cavity can then be computed using the virtual work principle $\Delta p \delta V_{\text {cav }}=\delta \epsilon_{e l}+\gamma \delta A_{\text {cav }}$, where we also introduced surface tension $\gamma$, and the volume $V_{\text {cav }}$ and area $A_{\text {cav }}$ of the cavity or drop. Using the spherical approximation for the cavity, estimating the virtual work from $\delta R$, one obtains the pressure inside the cavity

$$
p_{\text {cav }}=p_{\infty}+\frac{2 \gamma}{R}+\frac{\mu}{\xi^{2}} F^{\prime}(\xi),
$$

where $p_{\infty}$ is the atmospheric pressure far away from the droplet. Upon creasing $\xi=0.73$, using $\mu=\mu_{0}$, this results in $p_{\text {cav }}=-1.3 \mathrm{MPa}$, this pressure can be seen as a lower bound for the pressure upon which cavitation occurs. Under extreme deformations, PDMS (1:10) is better described using the Gent model [7], which takes into account the finite extensibility of polymer chains. However, for $\xi=0.73$ no significant difference in $F^{\prime}(\xi)$ is present for the two material models.

Suddenly, cavitation irrupts: A bubble appears and expands quickly, occupying almost the entire cavity previously occupied by water. Images of the early growth of such bubble are shown in the Supplemental Material [8]. Two distinct stages can be observed: At early times, the bubble grows at constant velocity [19] and fills up the cavity. By tracking the bubble growth velocity in that stage $\left(v_{i}\right)$ [8] we can probe the pressure of the liquid: Using $v_{i}=30 \mathrm{~m} / \mathrm{s}=\sqrt{-2 \Delta P /(3 \rho)}[20,21]$, we obtain $\Delta P=$ $-1.4 \mathrm{MPa}$, which is indeed consistent with the value estimated from creasing. Previous studies [7] also observed cavitation events in similar PDMS-formulation just before creasing. Although there is no general consensus regarding the critical pressure for cavitation in water, our values seem to yield somewhat lower tension as compared to other systems $[22,23]$. This difference can be explained by the fact that cavitation occurs in a more heterogeneous way in the PDMS matrix, due to its hydrophobic properties and porosity. In the later stage, the bubble has reached the cavity walls and the cavity expands to almost its original size. We will continue analyzing this last stage of growth. As can be seen in the Supplemental Material [8], the cavity is initially not perfectly spherical, as a result from the creasing event prior to cavitation. Therefore an effective radius is used to analyze the growth of this cavity.

The inset in Fig. 3 shows the growth of the elastic cavity as a function of time, for several initial droplet sizes. The main panel in Fig. 3 shows the result in dimensionless form, also including smaller initial droplet sizes. For all sizes we observe a power law for the growth stage, followed by (damped) oscillations and eventually a nearly static shape at $R / R_{0} \approx 0.9$. A few minutes after the cavitation event, the cavity will eventually grow to $R / R_{0} \approx 0.98$, practically recovering the initial droplet size. In Fig. 3, time is rescaled using $R_{0} / \sqrt{\mu / \rho}$, where $\sqrt{\mu / \rho}$ is the shear wave velocity. This appears to be the appropriate timescale for the cavity growth, showing that the dynamics emerge from an interplay between the cavity's inertia and the gel's elasticity. This is corroborated by the appearance of oscillations. However, they do not appear for all initial droplet sizes: Oscillations are not observed for the smallest droplet size $\left(R_{0}=172 \mu \mathrm{m}\right)$, suggesting a transition from overdamped to underdamped oscillations.

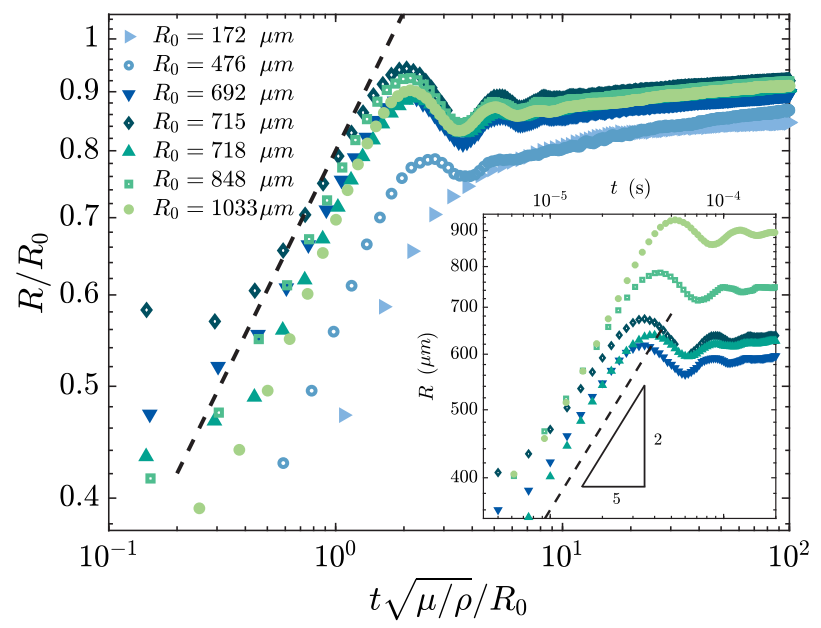

FIG. 3. Dynamics of the elastic cavity. The inset shows the growth of the cavity for different initial droplet radii. The main figure shows the nondimensionalized growth dynamics $R / R_{0}$ as a function of $t \sqrt{\mu / \rho} / R_{0}$ (with $\mu=1.7 \mathrm{MPa}$, see text). In both figures a power law of $2 / 5$ is displayed. The same legend applies for the two figures. The Supplemental Material [8] contains highspeed movies of the cavitation event and the subsequent cavity growth. 
Interestingly, all experiments performed for different initial droplet sizes yielded the same power law for the cavity growth (Fig. 3). This can be explained using the expression for the kinetic energy, which for an incompressible medium surrounding a spherical bubble reads $\epsilon_{\text {kin }}=2 \pi \rho R^{3} \dot{R}^{2}$. The term $\rho R^{3}$ represents the added mass of the cavity, and the same scaling should apply for cavities that are not perfectly spherical. As cavitation occurs, the elastic energy is quickly released, and assuming that the growth occurs at constant kinetic energy, we obtain $\dot{R}^{2} \sim 1 / R^{3}$, and thus $R \sim t^{2 / 5}$. Indeed, this is the scaling observed experimentally for all elastic cavities (Fig. 3). Fitting these experimental curves with $R=a t^{2 / 5}$ gives us the prefactor $a$, which allows us to extract a typical value for the kinetic energy $\epsilon_{\mathrm{kin}} \sim 0.25 \mathrm{~mJ}$. This can be compared to the elastic energy accumulated before cavitation, $0.77 \mathrm{~mJ}$ in the spherical approximation, which is indeed of the same order of magnitude. The difference between these values could be attributed to several factors as the remaining liquids kinetic energy, heat release and/or experimental inaccuracies. Note that the enthalpy required for the droplet to shrink is supplied as thermal energy and is in the order of $1 \mathrm{~J}$ (see the Supplemental Material [8] for a more detailed calculation). Consequently, only a small fraction of this energy is consumed to deform the elastic medium.

The scaling $R \sim t^{2 / 5}$ can be also obtained as the inertially dominated solution of the Rayleigh-Plesset equation [24]. We use here a modified Rayleigh-Plesset equation for cavities in elastic media given by

$\rho\left(R \ddot{R}+\frac{3}{2} \dot{R}^{2}\right)=p_{\text {cav }}-p_{\infty}-\frac{2 \gamma}{R}-\frac{\mu}{\xi^{2}} F^{\prime}(\xi)-\frac{4 \eta}{R} \dot{R}$,

where we introduced an effective viscosity $\eta$ of the surrounding medium $[21,25]$. The use of a damping of this "viscous" form can be derived only for small deformations, where the effective viscosity can be inferred from the loss modulus as $G^{\prime \prime}=\eta \omega$, see Supplemental Material at [8]. Note that considering the static solution $\dot{R}=0$ to this equation, we recover (1) for the pressure inside the cavity. Similar formulations of the Rayleigh-Plesset equation in elastic media have been previously used in the context of forced bubble oscillations [26-29].

We proceed by analyzing the cavity oscillations observed in Fig. 3 by considering small perturbations of the type $R(t)=R_{c}\left(1+\epsilon e^{i \omega t}\right)$, and linearizing (2), with $R_{c}$ the size of the elastic cavity. This gives an expression for the oscillation frequency,

$$
f \equiv \frac{\operatorname{Re}(\omega)}{2 \pi} \simeq \frac{1}{\pi} \sqrt{\frac{\mu}{\rho R_{c}^{2}}-\frac{\eta^{2}}{\rho^{2} R_{c}^{4}}},
$$

accounting for elasticity and viscous damping. Here, we omitted subdominant contributions due to the adiabatic

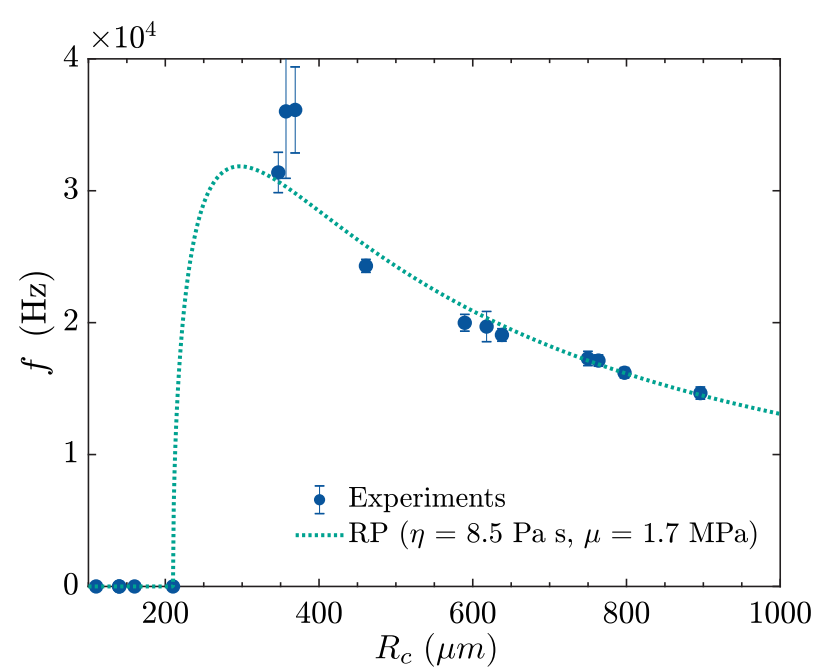

FIG. 4. Oscillation frequency of the elastic cavity. The experimental data is shown together with the solution of the RayleighPlesset equation (RP) including elasticity and viscosity [Eq. (3)]. The fitted values for the material parameters of the elastic medium, $\mu$ and $\eta$, are also given.

expansion of the vapor inside the cavity, and due to surface tension, which are respectively smaller by $10^{-1}$ and $10^{-3}$. The result (3) indeed predicts a transition from overdamped to underdamped oscillations, occurring at a critical drop radius $R^{*}=\eta / \sqrt{\mu \rho}$. In Fig. 4 we present our experimental measurements for the frequency, obtained by fitting $R(t)$ to exponentially damped oscillations. In this figure $f=0$ represents the overdamped case. Given that the bubble has not yet fully recovered, we defined the cavity radius $R_{c}$ as the mean of the maximum and minimum value of the first oscillation. To compare the experimental data to (3), we fit these data points using both the shear modulus $\mu$ and viscosity $\eta$ as adjustable parameters. The values found $(\mu=1.7 \mathrm{MPa}$ and $\eta=8.5 \mathrm{Pas})$ result in $R^{*}=210 \mu \mathrm{m}$. The fitted values for the shear modulus and viscosity match very well with available literature on rheological measurements of PDMS at high frequencies [30] and with our own measurements (see Supplemental Material at [8]). The excellent agreement in Fig. 4 shows that the oscillations are quantitatively captured by a viscoelastic RayleighPlesset-type model [31].

In summary, we have studied a cavitation process similar to that found in the cells of the fern sporangia. Our experiments capture and quantify the slow diffusive evaporation, which leads to a buildup of negative elastic pressure that eventually leads to a very fast cavitation. We provided detailed insight into the cavitation dynamics, which was accurately described by an inertial-(visco)elastic model. Understanding the mechanism by which certain plants are able to transform enthalpy into mechanical energy could lead a way to design synthetic materials able to perform similar fast motions making use of the this smart energy transformation found in nature. 
The authors acknowledge fruitful discussions with Benjamin Dollet, Sascha Hilgenfeldt, Javier RodríguezRodríguez, and Jens Eggers. We also thank one of the referees for suggesting the estimation of pressure from the initial bubble velocity. M. B. and A. M. acknowledge financial support from ERC (the European Research Council) Starting Grant No. 678573 NanoPacks. M. C. and J.H.S. acknowledge financial support from ERC Consolidator Grant No. 616918 Soft Wetting.

*m.a.bruning@utwente.nl

[1] J. Dumais and Y. Forterre, Annu. Rev. Fluid Mech. 44, 453 (2012).

[2] Y. Forterre, J. M. Skotheim, J. Dumais, and L. Mahadevan, Nature (London) 433, 421 (2005).

[3] X. Noblin, N. O. Rojas, J. Westbrook, C. Llorens, and J. Dumais, Science 335, 1322 (2012).

[4] T. D. Wheeler and A. D. Stroock, Nature (London) 455, 208 (2008).

[5] O. Vincent, P. Marmottant, P. A. Quinto-Su, and C. D. Ohl, Phys. Rev. Lett. 108, 184502 (2012).

[6] O. Vincent, P. Marmottant, S. R. Gonzalez-Avila, K. Ando, and C. D. Ohl, Soft Matter 10, 1455 (2014).

[7] M. P. Milner, L. Jin, and S. B. Hutchens, Soft Matter 13, 6894 (2017).

[8] See Supplemental Material at http://link.aps.org/supplemental/ 10.1103/PhysRevLett.123.214501 for rheological measurements of the PDMS-gel, energy estimations and images of the early stage growth of the bubble. It also includes movies of the long term evaporation process and high-speed movies of the cavitation event.

[9] D. Chen, L. Jin, Z. Suo, and R. C. Hayward, Mater. Horiz. 1, 207 (2014).

[10] S. Cai, K. Bertoldi, H. Wang, and Z. Suo, Soft Matter 6, 5770 (2010).

[11] F. Weiss, S. Cai, Y. Hu, M. Kyoo Kang, R. Huang, and Z. Suo, J. Appl. Phys. 114, 073507 (2013).

[12] L. Jin and Z. Suo, J. Mech. Phys. Solids 74, 68 (2015).

[13] F. Hamaguchi and K. Ando, Phys. Fluids 27, 113103 (2015).
[14] H. Gelderblom, A. G. Marín, H. Nair, A. Van Houselt, L. Lefferts, J. H. Snoeijer, and D. Lohse, Phys. Rev. E 83, 026306 (2011).

[15] S. J. Harley, E. A. Glascoe, and R. S. Maxwell, J. Phys. Chem. B 116, 14183 (2012).

[16] G. C. Randall and P. S. Doyle, Proc. Natl. Acad. Sci. U.S.A. 102, 10813 (2005).

[17] A. N. Gent and C. Wang, J. Mater. Sci. 26, 3392 (1991).

[18] A. N. Gent and P. B. Lindley, Proc. R. Soc. A 249, 195 (1959).

[19] The growth of this early stage bubble is linear, see Supplemental Material [8], showing that it obeys the steady-state solution of the unconfined Rayleigh-Plesset equation [20].

[20] O. Vincent and P. Marmottant, J. Fluid Mech. 827, 194 (2017)

[21] C. E. Brennen, Cavitation and Bubble Dynamics (Oxford University Press, New York, 1995).

[22] E. Herbert, S. Balibar, and F. Caupin, Phys. Rev. E 74, 041603 (2006).

[23] P. A. Quinto-Su and K. Ando, J. Fluid Mech. 733, R3 (2013).

[24] M. P. Brenner, S. Hilgenfeldt, and D. Lohse, Rev. Mod. Phys. 74, 425 (2002).

[25] V. N. Alekseev and S. A. Rybak, Acoust. Phys. 45, 535 (1999).

[26] R. Gaudron, M. T. Warnez, and E. Johnsen, J. Fluid Mech. 766, 54 (2015)

[27] J. B. Estrada, C. Barajas, D. L. Henann, E. Johnsen, and C. Franck, J. Mech. Phys. Solids 112, 291 (2018).

[28] A. Jamburidze, M. De Corato, A. Huerre, A. Pommella, and V. Garbin, Soft Matter 13, 3946 (2017).

[29] B. Dollet, P. Marmottant, and V. Garbin, Annu. Rev. Fluid Mech. 51, 331 (2019).

[30] V. Placet and P. Delobelle, J. Micromech. Microeng. 25, 035009 (2015).

[31] Note that in this model only viscous damping is taken into account, which can be verified by calculating the quality factors $Q_{\eta}$ and $Q_{\text {rad }}$, representing viscous damping and acoustic radiation. Using expressions for both quality factors as given in Ref. [20], results in $Q_{\eta}=O(1)$ and $Q_{\mathrm{rad}}=O(10)$, meaning that viscous dissipation is dominant in this system and thereby validating our approach. 